\title{
Regional origin and decrease of pain in patients with depressive symptoms under treatment with venlafaxine
}

\author{
Stefan Begré · Martin Traber · Martin Gerber • \\ Roland von Känel
}

Received: 8 December 2008/Accepted: 6 March 2009/Published online: 20 March 2009

(C) Springer-Verlag 2009

\begin{abstract}
Objective Patient's language, tradition, conventions, and customs may all determine integration into a society and are also part of the doctor-patient relationship that influences diagnostic and therapeutic outcome. Language barrier and sociocultural disparity of Eastern and Southern European patients may hamper recovery from pain and depression compared to Middle European patients in Switzerland.

Methods In a prospective naturalistic observational trial we investigated the influence of regional origin on treatment outcome in 420 pain sufferers with depressive symptoms from all over Switzerland who were treated with venlafaxine by 122 physicians in primary care. Physicians rated severity of depressive symptoms using the clinical global impression severity scale and pain intensity by means of visual analogue scales. We hypothesized that in Eastern and Southern European patients the magnitude of pain reduction under treatment with venlafaxine is less compared to Middle European patients.
\end{abstract}

\footnotetext{
S. Begré $(\bowtie) \cdot R$. von Känel

Division of Psychosomatic Medicine,

Department of General Internal Medicine,

University Hospital/Inselspital, 3010 Bern, Switzerland

e-mail: stefan.begre@insel.ch

R. von Känel

e-mail: roland.vonkaenel@insel.ch

M. Traber

Wyeth Pharmaceuticals AG,

Grafenauweg 10, Zug, Switzerland

e-mail: traberM@wyeth.com

M. Gerber

GEM Clinical Research Consulting M. Gerber,

Gewerbestrasse 6, Unterägeri/ZG, Switzerland

e-mail: mail@gem-crc.ch
}

Results Three months after study entry, Middle European patients were found to profit more from treatment with venlafaxine in terms of severity of depression and pain intensity than patients from Eastern Europe and Southern Europe.

Conclusion Regional origin may contribute to the magnitude of pain reduction in patients with depressive symptoms under treatment with venlafaxine. Our results provide a rational for care provider educational programs aimed at improving capacities in treating patients from different regional origin with psychosomatic complaints such as depression and comorbid pain.

Keywords Pain - Depression - Clinical practice . Migration $\cdot$ Switzerland

\section{Introduction}

A considerable number of immigrants from all over the world have settled in Switzerland since the 1960s; many of them recently immigrated from Balkan states and Turkey. Migration is associated with an increased risk of chronic pain [24]. Culture, ethnicity, and psychosocial environment may all have an important effect on pain perception and response $[10,28]$. The risk of endorsing depressed mood in patients with chronic pain is two to threefold increased $[4,12]$. Reported symptom clusters of depression differ in various cultures [28]. Pain has an important impact on response of depression to treatment in primary care [24], and negatively influences pain perception in the long-term $[2,30]$.

Treatment of chronic pain in patients with depressive symptoms is inherently difficult to provide [2, 3], cost intensive [14], and provided by various medical specialists 
amongst them primary care physicians (general practitioners and internists, and psychiatrists) [3]. Excessive pain behaviour may lead to unnecessary diagnostic testing and invasive procedures, and consequently iatrogenic complications and prolonged disability. In addition to rehabilitative and psychotherapeutic means, antidepressants may alleviate pain in depressed patients [19]. Venlafaxine is one of the most prescribed newer antidepressants and improves various types of pain $[1,2,11,16,22,25,27,31]$. Specifically, we recently showed that venlafaxine decreased pain in a population of depressed primary care patients, some of whom participated also in the present study [7]. Empirically, many physicians in Switzerland prescribe venlafaxine to their patients with chronic pain, particularly if the patients also show symptoms of depression. We recently found that venlafaxine decreased pain in depressed primary care patients who were in part also investigated in the present study.

Patient's language, tradition, conventions, and customs may all determine the doctor-patient relationship, thereby impacting diagnostic and therapeutic outcome. Doctors behave comparably less affectively when interacting with ethnic minority patients [26]. In turn, ethnic minority patients are less verbally expressive and they seem also to be less assertive and affective during the medical encounter relative to the average population [26]. Ethnic group identity is a predictor of locus of control which may considerably influence pain perception [6]. By reason of their language and sociocultural background, Eastern and Southern European patients may be less integrated into Swiss society than are immigrants from Middle Europe. In addition to sociocultural factors, the degree of integration into society affects the outcome of pain disorder in patients with a history of migration [24]. Many immigrants from Eastern Europe fled from the Balkan conflicts since 1991. Greater disease severity may relate to the experience of organized violence during warand war-like conflicts [13]. Negative affectivity in general has been proved to augment the burden of disease [21].

In this observational study, we therefore assumed that the magnitude of pain reduction in patients with depressive symptoms under treatment with venlafaxine differently relates to cultural regions of origin of patients. Naturalistic studies focussing on the treatment of patients with chronic pain and comorbid depressive symptomatology, investigating the impact of regional origin on treatment outcome in a primary care setting, are lacking. We hypothesized that in consequence of disparate sociocultural background, encountered challenges with integration into society, and language barrier in the doctor-patient relationship, Eastern European and Southern European patients would report greater pain intensity and less improvement of pain under treatment than those from Middle Europe. We statistically controlled for several covariates possibly affecting pain reduction, mainly the severity and duration of the disease, the venlafaxine dose prescribed at endpoint, and the duration of venlafaxine treatment.

\section{Methods}

Study design

The present study is part of a larger project which evaluated the therapeutic efficacy of venlafaxine in patients suffering pain and comorbid depressive symptoms [7]. For this purpose sales representatives invited 170 physicians from all regions in Switzerland among the medical specialities of psychiatry, general practice, and internal medicine. In Switzerland, venlafaxine is currently widely prescribed for this patient group. Therefore, study participation of physicians and their patients was not subject to incentives. Patients were included if they were newly prescribed venlafaxine (mostly within 2 weeks before visit 1). No other in- and exclusion criteria were applied. The proportion of contacted physicians working in the Italian, French, and Swiss German speaking parts of Switzerland was $1: 3: 6$. Totally 122 physicians participated in the study (accrual rate $72 \%$ ). Each practitioner could include a maximum of five patients and typically contributed four to five patients to the study. Patients' regional origin was asked and, after completion of data sampling, divided into three groups according to the respective Swiss publics opinion of national similarities across countries: (a) "Middle Europe" (Switzerland, Germany, Austria); (b) "Eastern Europe" (incl. Greece, Turkey, and other); and (c) "Southern Europe" (France, Italy, Spain, Portugal). This observational study was conducted in accordance with the Declaration of Helsinki and its amendments, considering the need for ethical approval and patient informed consent.

As detailed below, physicians determined diagnosis of depression by a regular clinical interview, estimated pain intensity using a visual analogue scale (VAS), and rated severity of depressive symptoms by the clinical global impression scale (CGI). Age, gender, duration of depression, and all clinically approved diagnoses for comorbid somatic and mental disorders (i.e. the first digit according to ICD-10) were listed. The use of opioid and non-opioid analgesics and the number of therapies in addition to those targeting depression and pain were also recorded. Data were obtained at study entry. Venlafaxine dosages and CGI and VAS scores were also obtained at two follow-up visits intended to take place at 1 and 3 months after study entry. 
Study participants

Of totally 601 patients, participating in the parent study, we report here on 420 patients who had conclusive information available for CGI severity score and pain intensity scores at study entry (also termed initial visit or visit 1), at the first follow-up visit (also termed visit 2), and at the second follow-up visit (also termed endpoint visit or visit 3), and for the dosage of venlafaxine prescribed at visit 3 (according to available formulas of the substance and commonly prescribed dosages coded as $1=0 \mathrm{mg}, 2=1-$ $75 \mathrm{mg}, 3=76-150 \mathrm{mg}$, or $4=151-450 \mathrm{mg}$ ), and time between visit 1 and visit 3 in days. If data from visit 3 were missing, the last observation from visit 2 was carried forward to the endpoint (i.e. data of visit 2 were used as endpoint measures for visit 3). Information was also required to be complete for regional origin (coded as $1=$ Middle Europe, 2 = Eastern Europe incl. Turkey, or $3=$ Southern Europe), speciality of the treating physician (coded as $1=$ psychiatrist (PSY), $2=$ general practitioner (GP), or $3=$ internist (IM)), gender, age, the number of ICD-10 diagnosis other than related to pain or depression (coded as 0,1 , or $\geq 2$ diagnoses), duration of depressive symptomatology, and time between visits allowing for full analysis of covariance (ANCOVA). The use of prescribed non-opioids, low potent opioids (i.e. tramadol, tilidine, dihydrocodeine), and high-potent opioids (i.e. morphine, buprenorphine, fentanyl, hydromorphone, oxycodone) (all coded as $0=$ no or $1=$ yes), other therapies for pain or depression (coded as $0=$ no or $1=$ yes), duration of depressive symptoms in months, and duration of pain in months were all assessed by physician report.

\section{Psychometric assessment}

\section{Diagnosis of depression}

Within the scope of the common diagnostic procedure in Swiss primary care referring to conversant diagnostic criteria for depression, physicians established the diagnosis of depression based on history taking, the clinical interview, and clinical impression of the patient. By reason of their limited time resources within this naturalistic observational study, they did not apply a structured diagnostic interview tool to establish a diagnosis of depression.

\section{$C G I$}

CGI is a three-item scale used to assess treatment response and can also be used for measuring the severity of a depressive state [5]. Severity of Illness (item 1) is rated on a seven-point scale $(1=$ normal to $7=$ extremely ill $)$; global improvement (item 2) is rated on a seven-point scale
$(1=$ very much improved to $7=$ very much worse $)$; and the efficacy index (item 3 ) is rated on a four-point scale (from 'none' to 'outweighs therapeutic effect') [15].

\section{$V A S$}

Pain intensity was estimated in terms of the five main body regions (i.e. back, extremities, head, abdomen, and chest) by a VAS ranging from 0 (no pain) to 10 (extreme pain). Since many patients suffering depressive symptomatology and pain report pain in more than one region of the body a total pain severity score was computed by adding up the VAS scores of the five individual pain regions.

\section{Statistical analysis}

We used SPSS 13.0 for Windows to analyse the data. A normal distribution could not be achieved for measures of depression duration, pain duration, and time from visit 1 to visit 3 by any transformation procedure. For these measures we formed a categorical variable with each category including a similar number of subjects to be used as a covariate in repeated measures analyses of covariance (RM ANCOVA). The categories were for the duration of depression: (1) 0-1 month $(n=108), \quad(2) \quad 2-7$ months $(n=105)$, (3) 8-32 months $(n=101)$, and (4) 33-377 months $(n=106)$; for the duration of pain: (1) $0-4$ months $(n=112)$, (2) 5-18 months $(n=99)$, (3) 19-53 months ( $n=105)$, and (4) 54-424 months $(n=104)$; and for the time from visit 1 to visit 3: (1) 7-87 days $(n=107)$, (2) 88-94 days $(n=106)$, (3) 95-106 days $(n=102)$, and (4) $107-413$ days $(n=105)$.

All tests were two-tailed and the level of significance was set at $P<0.05$. Pearson chi-square test, KruskalWallis test, and one-way ANOVA were used to examine differences between regional origin in terms of categorical and normally or non-normally distributed continuous variables where appropriate. Changes in the CGI severity and pain scores over time were examined using RM ANCOVA with time (visit 1, visit 2, visit 3 ) as the withinsubjects factor and with regional origin (Middle Europe, Eastern Europe, Southern Europe) as the betweensubjects factor controlling for CGI severity score at visit 1 , the respective pain scores at visit 1 , venlafaxine dosage at endpoint, and the time elapsed between visit 1 and visit 3. Demographic and medical covariates were gender, age, physician speciality, use of non-opioid analgesics, use of low and high-potent opioids combined, other therapies for pain and depression, number of ICD-10 diagnosis other than related to pain and depression, duration of depressive symptomatology, and duration of pain. Fisher's least significant difference was used for post hoc testing. 


\section{Results}

Patient characteristics

The subsample of 420 patients investigated here did not significantly differ from the total sample of 601 patients at visit 1 in terms of origin, age, severity and duration of depression, duration of pain, the number of ICD-10 diagnoses other than depression and pain, the use of prescribed analgesics (non-opioids, low- and high-potent opioids), and other therapies for pain or depression. The subsample comprised a lower proportion of women; there were no gender differences between sample and subsample with regard to age and the medical characteristics reported above.

Patients were from Middle Europe in 58\%, from Eastern Europe incl. Turkey in 26\%, and from Southern Europe in $16 \%$. Table 1 shows the differences in demographic and medical characteristics of the 420 patients studied by regional origin.

Change in severity of depression

Figure 1 shows the interaction between time and regional origin for the change in the CGI severity score between visits 1 and $3\left(F_{2,406}=14.7, P<0.001\right)$. The magnitude of the decrease in the CGI score over time was greater in patients from Middle Europe than in those from the Eastern Europe $(P<0.001)$ and in those from Southern Europe $(P=0.001)$, whereas no significant difference was observed between Eastern European and Southern European patients.

\section{Change in pain severity}

There was an interaction between time and regional origin for a reduction in total pain severity from visit 1 to visit $3\left(F_{2,405}=5.3, P=0.005\right.$; Fig. $\left.2 \mathrm{a}\right)$. The magnitude of the decrease in total pain intensity over time was greater in patients from Middle Europe than in those from the Eastern Europe $(P<0.001)$ and from Southern Europe $(P=0.006)$; pain reduction was not significantly different between Eastern European and Southern European patients.

Separate analyses for the individual pain regions revealed interactions between time and regional origin for a decrease in severity of back pain $\left(F_{2,405}=9.5, P<0.001\right.$; Fig. 2b), extremities pain $\left(F_{2,405}=7.4, \quad P=0.001\right.$; Fig. 2c), and pain localised to the head $\left(F_{2,405}=5.6\right.$,

Table 1 Characteristics of the 420 patients by categories of regional origins

\begin{tabular}{|c|c|c|c|c|}
\hline & Middle Europe $(n=243)$ & Eastern Europe $(n=108)$ & Southern Europe $(n=69)$ & $P$ value \\
\hline Women/men & $63 / 37$ & $57 / 43$ & $61 / 39$ & 0.567 \\
\hline Age (years) & $54.5 \pm 16.1$ & $43.7 \pm 8.8$ & $50.2 \pm 10.7$ & $<0.001$ \\
\hline Physician speciality (PSY, GP, IM) & $18 / 59 / 23$ & $42 / 39 / 19$ & $20 / 60 / 20$ & $<0.001$ \\
\hline Duration of depression (months) & $7(1-41)$ & $7(2-23)$ & $9(1-40)$ & 0.827 \\
\hline Duration of pain (months) & $15(3-52)$ & $22(6-47)$ & $34(7-66)$ & 0.004 \\
\hline Non-opioid analgesics (years/n) & $86 / 14$ & $86 / 14$ & $85 / 15$ & 0.993 \\
\hline Low potent opioids (years/n) & $23 / 77$ & $30 / 70$ & $26 / 74$ & 0.417 \\
\hline High-potent opioids (years/n) & $5 / 95$ & $1 / 99$ & $6 / 94$ & 0.153 \\
\hline Other therapies ${ }^{\mathrm{a}}$ & $49 / 51$ & $25 / 75$ & $45 / 55$ & $<0.001$ \\
\hline ICD-10 diagnosis $(0,1 \geq 2)^{\mathrm{b}}$ & $37 / 33 / 30$ & $59 / 31 / 10$ & $43 / 35 / 22$ & $<0.001$ \\
\hline CGI severity score at visit $1(1-7)$ & $4.5 \pm 0.9$ & $4.9 \pm 0.8$ & $4.7 \pm 0.9$ & 0.001 \\
\hline Total pain at visit $1(0-50)$ & $13.9 \pm 7.5$ & $18.0 \pm 7.8$ & $19.2 \pm 8.8$ & $<0.001$ \\
\hline Back pain at visit $1(0-10)$ & $4.8 \pm 2.4$ & $6.5 \pm 2.9$ & $6.5 \pm 2.6$ & $<0.001$ \\
\hline Extremities pain at visit $1(0-10)$ & $3.5 \pm 3.4$ & $4.5 \pm 3.4$ & $5.0 \pm 3.4$ & 0.001 \\
\hline Head pain at visit $1(0-10)$ & $3.1 \pm 3.1$ & $4.8 \pm 3.1$ & $4.3 \pm 3.3$ & $<0.001$ \\
\hline Abdomen pain at visit $1(0-10)$ & $1.4 \pm 2.5$ & $0.8 \pm 1.9$ & $1.7 \pm 2.7$ & $<0.001$ \\
\hline Thoracic pain at visit $1(0-10)$ & $1.0 \pm 2.1$ & $1.3 \pm 2.2$ & $1.8 \pm 2.3$ & $<0.001$ \\
\hline Venlafaxine dose at visit $3(\mathrm{mg})$ & $137.4 \pm 64.0$ & $160.7 \pm 76.0$ & $145.8 \pm 71.3$ & 0.020 \\
\hline Time between visit 1 and 3 (days) & $94(87-109)$ & $93(84-104)$ & $96(88-111)$ & 0.637 \\
\hline
\end{tabular}

Values are given as mean $\pm \mathrm{SD}$, median (with inter-quartile range), or percentage. Analyses used either Pearson chi-square test, Kruskal-Wallis test, or one-way analysis of variance

$C G I$ clinical global impression, GP general practice, $I C D$ international classification of diseases, $I M$ internal medicine, $P S Y$ psychiatry

${ }^{\text {a }}$ Other than for pain or depression

b Other than related to pain or depression 


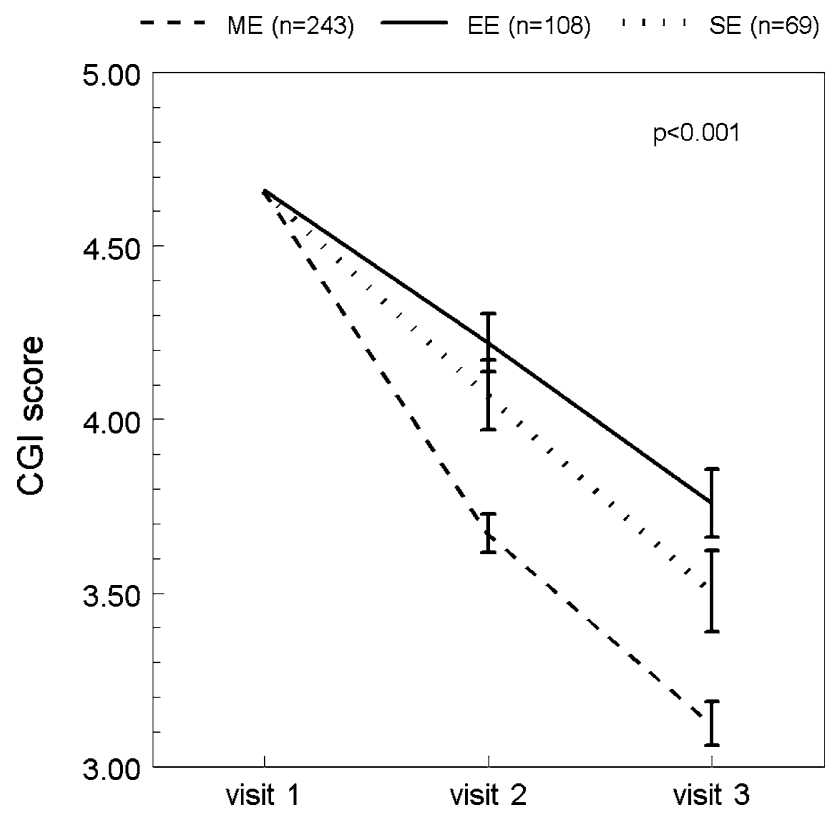

Fig. 1 The graph shows the significantly different mean \pm SEM decrease in the clinical global impression (CGI) severity score over time between regional origins after adjustment for covariates (cf. text). The CGI severity score decreased significantly less in patients from Eastern Europe (EE) and from Southern Europe (SE) compared to those from Middle Europe (ME)

$P=0.004$; Fig. $2 \mathrm{~d})$. Reduction in pain severity localised to the back $(P<0.001)$ and to the head $(P \leq 0.002)$ was greater in patients from Middle Europe than in those from the Eastern Europe and from Southern Europe. In addition, reduction in extremities pain was greater in patients from Middle Europe than in those from the Eastern Europe $(P<0.001)$. There were no significant changes over time in pain severity localised to the abdomen and thorax. Patients from the Eastern Europe and from Southern Europe did not significantly differ in changes of any pain score.

\section{Discussion}

Supporting our hypothesis, Eastern European and Southern European patients showed less therapeutic benefit in terms of a decrease in the CGI severity score and overall pain intensity compared to their Middle European counterparts when controlling for a series of covariates possibly affecting severity of depression, pain perception and chronic manifestation. The magnitude of the decrements in the CGI and VAS scores was clinically meaningful. One point decrease from 4 to 3 on the CGI indicates change from moderate to mild impairment, whereas the decrement of two points in the VAS score is considered to be a relevant pain reduction [31].
We offer three possible explanations for these finding, even if these not ultimately derive from our results. First, successful treatment of chronic pain is based on a multilevel approach that confers various rehabilitative and psychotherapeutic means in addition to the expected benefits of drug treatment. Therefore, the doctor-patient relationship may play an important role in delivering advice, and in establishing diagnostic and therapeutic procedures. By reason of language difficulties and disparate sociocultural background, the doctor-patient relationship might have been hampered mostly in Eastern European patients. Second, psychosocial conditions in the host country affect the outcome of pain disorders. Eastern European patients in Switzerland have comparably less accomplished integration because of language difficulties, sociocultural disparity, and most recent migration. It could previously be shown that psychosocial background $[6,13$, 26] and the degree of integration into society [24] may influence pain behaviour. Third, in this observational study, we did not differ between distinct kinds of chronic pain. But, psychiatrists treated the highest percentage of Eastern European patients (42\%) compared to general practitioners (39\%) and internists (19\%). According to the Swiss care providing system, psychiatrists are referred patients with chronic pain after these have undergone treatment by general practitioners and internists. Therefore, even if this reasoning remains speculative, we may suggest that Eastern European patients suffer more from somatoform pain than the two other patient groups because somatoform pain in general is difficult to treat, especially by internists who received comparably few training in the clinical management of psychiatric disorders. Supporting this third explanation, compared to Middle European and Southern European patients, Eastern European patients were younger, showed higher intensity of depressive symptoms, endorsed a comparably lower number of diagnoses in addition to depression and pain, and received a lower number of other therapies for depression and chronic pain. In contrast, relatively more frequent care to Middle European and Southern European patients was provided by a general practitioner. The latter two patient groups received also more frequently therapies for conditions above and beyond depression and pain.

In terms of pain intensities of different body regions at endpoint, Middle European patients suffered less from back and head pain than Eastern and Southern European patients, and Middle European patients had lower intensity of extremities pain than Southern European patients. To interpret these data, we suppose a relation to a different way of pain behaviour and expression, and doctor-patient interaction as previously shown $[10,13,24,26,28]$. There were no significant changes over the study period of 3 months in pain severity localised to the abdomen and 
Fig. 2 The graphs show the significantly different mean \pm SEM decreases in pain intensity scores over time between regional origins after adjustment for covariates (cf. text). Intensity of total pain (a), back pain (b), and head pain (d) decreased all significantly less in patients from Eastern Europe (EE) and from Southern Europe (SE) compared to patients from Middle Europe (ME).

Extremities pain decreased significantly less in Eastern European patients than in Middle European patients (c)
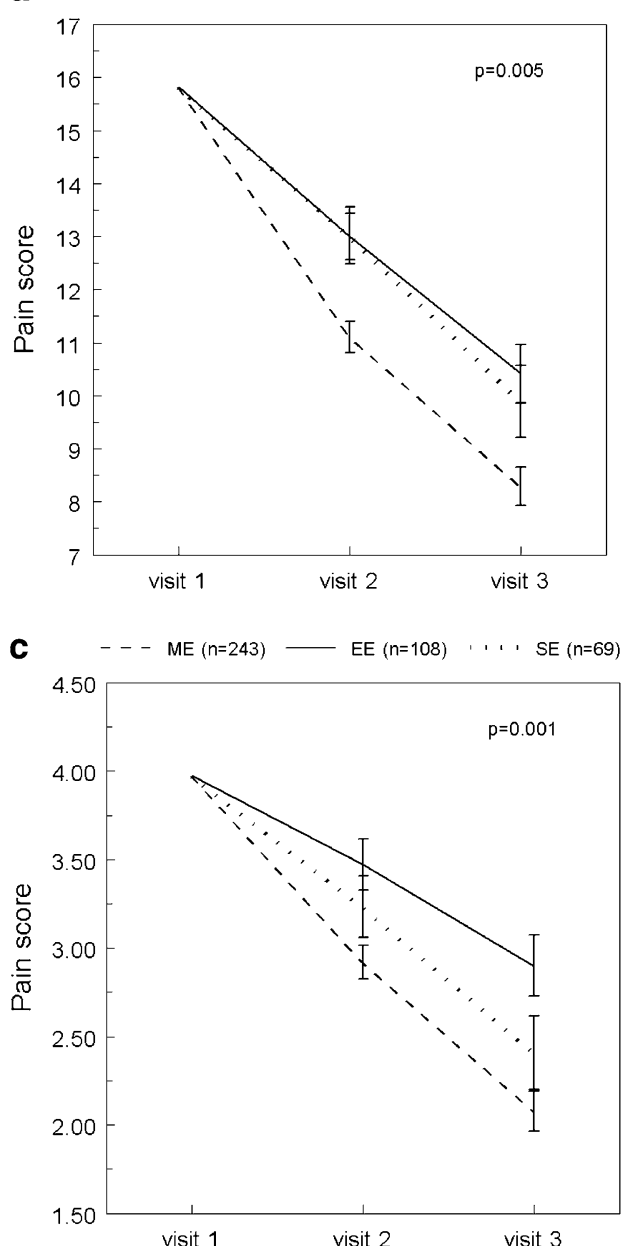

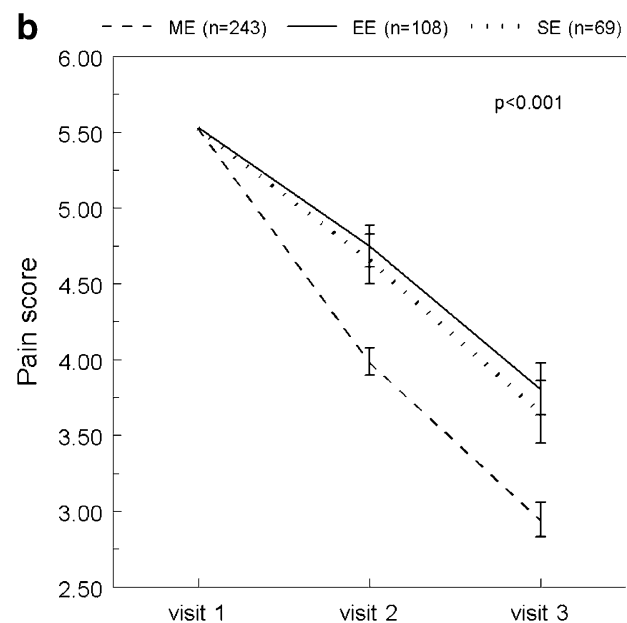

d

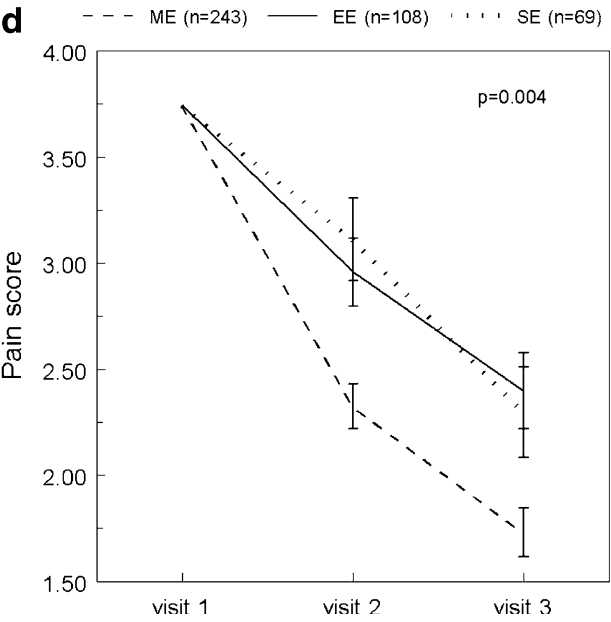

thorax between the three patients groups. This could be explained by the comparably low levels of abdominal and thoracic pain in our sample at study entry and may not reflect cultural differences in pain experience or psychosocial interactions with the host country. In fact, only about one-third of all patients reported thoracic and abdominal pain with mostly minimal intensity.

Even if Eastern and Southern European patients, in accordance with greater severity of pain and depressive symptoms at study entry, received on average slightly higher mean doses of venlafaxine at endpoint, they improved less in terms of depression and pain compared to Middle European patients. There was a strong relationship between depression and pain improvement. However, even when taking into account depression and pain at study entry, Eastern and Southern European patients still improved less during treatment with venlafaxine. We did not monitor compliance based on plasma levels of venlafaxine or compliance questionnaires. Yet, during the study, 3 of 108 patients from Eastern Europe and 3 of 69 patients from Southern Europe dropped out, whereas only 217 of 243 Middle European patients could be followed up to visit
3. Therefore, worse compliance in Eastern and Southern European compared to Middle European patients as a possible confounder seems unlikely. In sum, neither compliance during the study nor severity of depression or pain at study entry seem to explain our finding of a less favourable outcome in Eastern and Southern European compared to Middle European patients. Instead, based on our consideration above and as delineated in the introduction, we interpret our findings rather to be due to certain other factors. These might relate to somatoform pain that is particularly difficult to treat and may be more prevalent in Eastern and Southern European patients, as well as to unfavourable ethnic and cultural factors which impact the doctor-patient communication $[6,24,26]$ at the beginning and during treatment such as to worsen outcome $[8,9,13]$. In addition underdose of antidepressants in general was previously reported in primary care settings $[18,29]$ and might also play a particular role in patients from Eastern and Southern Europe.

Our data should be interpreted within the particular limitations of the study design. A psychiatric diagnosis of depression was not formally established by a structured 
interview. Also, the number of ICD-10 diagnoses is a rather crude measure to reliably account for the "true" comorbidity. Furthermore, we did not consider duration of stay and family relations in the host country, socioeconomic differences, and treatment for depression and pain prior to inclusion in the study and during the observational phase besides venlafaxine. Overall, Eastern European patients were more severely ill at study entry than Middle European patients but experienced less benefit from treatment. This is particularly intriguing since Eastern European patients received, on average, the highest dose of venlafaxine in accordance with the state of the art suggesting dose augmentation with increasing severity of the disease [20,23], even if dosage may still be too low for causing a satisfying effect. This finding has to be interpreted bearing in mind that our study was observational and lacked a control group treated with an antidepressant other than venlafaxine.

The pragmatic study design represents the unequal caring situation for patients with chronic pain and comorbid depressive symptomatology in Switzerland. Our results are based on a sample size recruited from all Swiss regions and by the physician specialities primarily involved in management of these patient groups. Our data could particularly encourage primary care providers to deal with these, for the healthcare system, challenging and costly patients, who may draw at least equally strong health benefit from both somatic primary care as from psychiatry. Our results provide a rational for education programs targeting at care providers who are involved in the treatment of patients from different regional origins with psychosomatic complaints [17].

\section{Conclusion}

Bearing in mind the study limitations, we may conclude that regional origin may contribute to the magnitude of pain reduction in patients with depressive symptoms under treatment with venlafaxine. Even though Eastern European patients were more severely ill than Middle European patients at study entry, and Eastern European and Southern European patients had greater pain than Middle European patients, Eeastern European and Southern European patients experienced less benefit from treatment compared to their Middle European counterparts.

Acknowledgment We are grateful to Wyeth Pharmaceuticals AG, Switzerland for providing its data pool to perform this study.

\section{Reference List}

1. Adelman LC, Adelman JU, Von Seggern R, Mannix LK (2000) Venlafaxine extended release (XR) for the prophylaxis of migraine and tension-type headache: a retrospective study in a clinical setting. Headache 40:572-580

2. Arnow BA, Hunkeler EM, Blasey CM, Lee J, Constantino MJ, Fireman B, Kraemer HC, Dea R, Robinson R, Hayward C (2006) Comorbid depression, chronic pain, and disability in primary care. Psychosom Med 68:262-268

3. Bair MJ, Robinson RL, Eckert GJ, Stang PE, Croghan TW, Kroenke K (2004) Impact of pain on depression treatment response in primary care. Psychosom Med 66:17-22

4. Bair MJ, Robinson RL, Katon W, Kroenke K (2003) Depression and pain comorbidity: a literature review. Arch Intern Med 163:2433-2445

5. Bandelow B, Baldwin DS, Dolberg OT, Andersen HF, Stein DJ (2006) What is the threshold for symptomatic response and remission for major depressive disorder, panic disorder, social anxiety disorder, and generalized anxiety disorder? J Clin Psychiatry 67:1428-1434

6. Bates MS, Edwards WT, Anderson KO (1993) Ethnocultural influences on variation in chronic pain perception. Pain 52: 101-112

7. Begré S, Traber M, Gerber M, von Kaenel R (2008) Change in pain severity with open label venlafaxine use in patients with a depressive symptomatology: an observational study in primary care. Eur Psychiatry 23(3):178-186

8. Cutrona CE, Russell DW, Brown PA, Clark LA, Hessling RM, Gardner KA (2005) Neighborhood context, personality, and stressful life events as predictors of depression among African American women. J Abnorm Psychol 114:3-15

9. Eisenman DP, Gelberg L, Liu H, Shapiro MF (2003) Mental health and health-related quality of life among adult Latino primary care patients living in the United States with previous exposure to political violence. JAMA 290:627-634

10. Fillingim RB (2005) Individual differences in pain responses. Curr Rheumatol Rep 7:342-347

11. Freeman EW, Rickels K, Yonkers KA, Kunz NR, McPherson M, Upton GV (2001) Venlafaxine in the treatment of premenstrual dysphoric disorder. Obstet Gynecol 98:737-744

12. Geerlings SW, Twisk JW, Beekman AT, Deeg DJ, van Tilburg W (2002) Longitudinal relationship between pain and depression in older adults: sex, age and physical disability. Soc Psychiatry Psychiatr Epidemiol 37:23-30

13. Gilgen D, Gross CS, Maeusezahl D, Frey C, Tanner M, Weiss MG, Hatz C (2002) Impact of organized violence on illness experience of Turkish/Kurdish and Bosnian migrant patients in primary care. J Travel Med 9:236-240

14. Greenberg PE, Leong SA, Birnbaum HG, Robinson RL (2003) The economic burden of depression with painful symptoms. J Clin Psychiatry 64(Suppl 7):17-23

15. Guy W (1976) ECDEU assessment manual for psychopharmacology (rev edn). National Institute for Mental Health, Rockville

16. Karp JF (2004) Venlafaxine XR and chronic pelvic pain syndrome. J Clin Psychiatry 65:880-881

17. Leyer EM (1990) Hidden interpersonal structures in medical and psychotherapy interaction with foreign patients-presented and discussed with the example of a Turkish patient with chronic pain. Psychother Psychosom Med Psychol 40: 423-431

18. Lin EH, Katon WJ, Simon GE, Von Korff M, Bush TM, Walker EA, Unutzer J, Ludman EJ (2000) Low-intensity treatment of depression in primary care: is it problematic? Gen Hosp Psychiatry $22: 78-83$

19. Lynch ME (2001) Antidepressants as analgesics: a review of randomized controlled trials. J Psychiatry Neurosci 26:30-36

20. Mbaya P (2002) Safety and efficacy of high dose of venlafaxine $\mathrm{XL}$ in treatment resistant major depression. Hum Psychopharmacol 17:335-339 
21. McCrae JD, Lumley MA (1998) Health status in sickle cell disease: examining the roles of pain coping strategies, somatic awareness, and negative affectivity. J Behav Med 21:35-55

22. Reuben SS (2005) The prevention of post-surgical neuralgia. Pain 113:242-243

23. Rudolph RL, Fabre LF, Feighner JP, Rickels K, Entsuah R, Derivan AT (1998) A randomized, placebo-controlled, doseresponse trial of venlafaxine hydrochloride in the treatment of major depression. J Clin Psychiatry 59:116-122

24. Sabbioni ME, Eugster S (2001) Interactions of a history of migration with the course of pain disorder. J Psychosom Res 50:267-269

25. Sayar K, Aksu G, Ak I, Tosun M (2003) Venlafaxine treatment of fibromyalgia. Ann Pharmacother 37:1561-1565

26. Schouten BC, Meeuwesen L (2006) Cultural differences in medical communication: a review of the literature. Patient Educ Couns 64:21-34
27. Songer DA, Schulte H (1996) Venlafaxine for the treatment of chronic pain. Am J Psychiatry 153:737

28. Ulusahin A, Basoglu M, Paykel ES (1994) A cross-cultural comparative study of depressive symptoms in British and Turkish clinical samples. Soc Psychiatry Psychiatr Epidemiol 29:31-39

29. Weilburg JB, O'Leary KM, Meigs JB, Hennen J, Stafford RS (2003) Evaluation of the adequacy of outpatient antidepressant treatment. Psychiatr Serv 54:1233-1239

30. Williams LS, Jones WJ, Shen J, Robinson RL, Kroenke K (2004) Outcomes of newly referred neurology outpatients with depression and pain. Neurology 63:674-677

31. Yucel A, Ozyalcin S, Koknel TG, Kiziltan E, Yucel B, Andersen OK, Arendt-Nielsen L, Disci R (2005) The effect of venlafaxine on ongoing and experimentally induced pain in neuropathic pain patients: a double blind, placebo controlled study. Eur J Pain 9:407-416 